\title{
Global Stability of a SVEIR Epidemic Model: Application to Poliomyelitis Transmission Dynamics
}

\author{
L. N. Nkamba', ${ }^{1,}$, J. M. Ntaganda3 ${ }^{3}$, H. Abboubakar ${ }^{2,4,5}$, J. C. Kamgang,6, Lorenzo Castelli ${ }^{7}$ \\ ${ }^{1}$ Department of Mathematics, Higher Teacher Training College, University of Yaoundé I, Yaoundé, Cameroon \\ ${ }^{2}$ Laboratoire d'Analyse, Simulation et Essai (LASE), Ngaoundéré, Cameroon \\ ${ }^{3}$ Department of Mathematics, School of Science, College of Science and Technology, University of Rwanda, Butare, Rwanda \\ ${ }^{4}$ Laboratoire de Mathématiques Expérimentales (LAMEX), Ngaoundere, Cameroon \\ ${ }^{5}$ Department of Computer Engineering, University of Ngaoundéré, UIT, Ngaoundéré, Cameroon \\ ${ }^{6}$ Department of Mathematics and Computer Science, ENSAI-University of Ngaoundere, Ngaoundéré, Cameroon \\ ${ }^{7}$ DIA-University of Trieste, Trieste, Italy \\ Email: ^Inkague@gmail.com, jeanmariefriends@yahoo.fr, abboubakarhamadjam@yahoo.fr, h.abboubakar@gmail.com, \\ jckamgang@gmail.com, castelli@units.it
}

How to cite this paper: Nkamba, L.N., Ntaganda, J.M., Abboubakar, H., Kamgang, J.C. and Castelli, L. (2017) Global Stability of a SVEIR Epidemic Model: Application to Poliomyelitis Transmission Dynamics. Open Journal of Modelling and Simulation, 5, 98112.

http://dx.doi.org/10.4236/ojmsi.2017.51008

Received: November 12, 2016

Accepted: January 15, 2017

Published: January 18, 2017

Copyright (c) 2017 by authors and Scientific Research Publishing Inc. This work is licensed under the Creative Commons Attribution International License (CC BY 4.0).

http://creativecommons.org/licenses/by/4.0/

\begin{abstract}
The lack of treatment for poliomyelitis doing that only means of preventing is immunization with live oral polio vaccine (OPV) or/and inactivated polio vaccine (IPV). Poliomyelitis is a very contagious viral infection caused by poliovirus. Children are principally attacked. In this paper, we assess the impact of vaccination in the control of spread of poliomyelitis via a deterministic SVEIR (Susceptible-VaccinatedLatent-Infectious-Removed) model of infectious disease transmission, where vaccinated individuals are also susceptible, although to a lesser degree. Using LyapunovLasalle methods, we prove the global asymptotic stability of the unique endemic equilibrium whenever $\mathcal{R}_{\text {vac }}>1$. Numerical simulations, using poliomyelitis data from Cameroon, are conducted to approve analytic results and to show the importance of vaccinate coverage in the control of disease spread.
\end{abstract}

\section{Keywords}

Deterministic SVEIR Model, Poliomyelitis, Imperfect Vaccine, Direct Lyapunov Method, Equilibrium States, Global Stability

\section{Introduction}

In the 70s, having noticed that five million children died every year further to an avoidable disease by the vaccination like poliomyelitis, the WHO introduced the Global Immunization Vision and Strategy (GIVS). Poliomyelitis has been eliminated in the 
most of countries, but recently we observe the upsurge of infectious in some countries [1]. Since October 2013, Cameroon is classified by the WHO as the exporting country of the poliovirus [2]. Poliomyelitis is an acute and sometimes devastating viral disease very contagious caused by poliovirus. Human is the only natural host for poliovirus [3]. Children are principally attacked. Poliovirus is predominantly transmitted via mother and food contaminated. In the most of case, infection is asymptomatic but the persons infected can transmit disease via their feces [4]. When a susceptible is exposed to infection by a virulent poliovirus, we can observe few days or few weeks three types of responses (minor illness, aseptic meningitis, and paralytic poliovirus). In case of minor illness, after 3 - 5 days, symptoms can be slight, fever, tiredness, headache, sore throat and vomiting. In the minor illness, the patient recovers in a few days 24 to 72 hours. In the case of non paralytic poliomyelitis in addition in some of minor illness signs and symptoms includes stiffness and pain in the back of neck. In the past days of illness, healing will rapid and complete. In the paralytic poliomyelitis, the predominant damage is flaccid paralysis resulting from lower motor neurons damage. The maximal recovery usually occurs after 6 months, but residuals paralysis lasts much longer. There does not exist a specific treatment for poliomyelitis although improved sanitation and hygiene help to limit the spread of poliovirus. The only specific means of preventing polio is immunization with live polio vaccine (OPV) or/and inactivated polio vaccine (IPV) [5] [6] [7] [8].

As part of the necessary multi-disciplinary research approach, mathematical models have been extensively used to provide a framework for understanding of poliomyelitis transmission dynamics and the best strategies to control the spread of infection in the human population. In the literature, considerable work can be found on the mathematical modeling of poliomyelitis [9]-[18]. Some of these works refer to vaccination as polio control mechanism [9] [12] [17] [18], using a standard SEIR model [19].

Some SVEIR models are used to assess the potential impact of an imperfect SARS vaccine like SARS vaccine [20], Hepatitis B vaccine [21], Tuberculosis vaccine [22], HIV vaccine [23] [24], to mention only these four diseases. From a mathematical point of view, to show the global asymptotic stability of equilibrium points in general, and especially, the global asymptotic stability of the endemic equilibrium, is not an easy task. This requires, in most cases, the use of several different techniques, such as the theory of compound matrix [25] [26], the comparison theorem [27], or the use of Lyapunov functions associated with the Lassalle invariance principle [28], to name a few techniques commonly used by authors. For example, in [20], the authors used compound matrix techniques to show the global stability of the endemic equilibrium under some constraints on the parameters of the system. Huiming Wei et al. [29] proposed an SVEIR model with time delay, and analyzed the dynamic behavior under pulse vaccination. Using comparison theorem, they showed that the infection-free periodic solution is globally attractive. Yu Jiang et al. [30] modified that model by adding saturation incidence, and used too the comparison theorem to show the global stability of "infection-free" periodic solution.

In this paper, we study the impact of vaccination in the control of poliomyelitis spread via an SVEIR model of infectious disease transmission. Individuals are classified 
as one of susceptible $S$, vaccinated $V$, exposed $E$, infectious $I$, or recovered $R$. The model is based on a standard SEIR model [19], but allows that susceptible individuals may be given an imperfect vaccine that reduces their susceptibility to the disease. Since we consider a leaky vaccine, the V-compartment of vaccinated individuals is considered as a susceptible compartment, and thus we are dealing with a differential susceptibility system with bilinear mass action as in Hyman and Li [31]. However, we include one-way flow between these two compartments due to vaccination making the model studied here distinct from the model in [31]. For the case where the basic reproduction number is less than one, the global stability of the disease-free equilibrium has been shown by Gumel et al. in 2006 [20]. However, the global dynamics when the basic reproduction number is greater than one have not been resolved before. By allowing different death rates for each of the compartments, the model studied in this paper is slight generalization of the model studied in [20]. Using Lyapunov-LaSalle methods, we fully resolve the global dynamics of the model for the full parameter space. We demonstrate that the model exhibits threshold behavior with a globally stable disease-free equilibrium if the basic reproduction number is less than unity and alobally stable endemic equilibrium if the basic reproduction number is greater than unity. Thus, we also fully resolve the global dynamics for the model studied in [20].

In order to study the stability of a positive endemic equilibrium state, we use Lyapunov's direct method and LaSalle's Invariance Principle with a Lyapunov function of the form:

$$
V\left(x_{1}, x_{2}, \cdots, x_{n}\right)=\sum_{i} A_{i}\left(x_{i}-x_{i}^{*} \ln x_{i}\right)
$$

where $A_{1}, \cdots, A_{n}$ are constants, $x_{i}$ is the population of th compartment and $x_{i}^{*}$ is the equilibrium level. Lyapunov functions of this type have also proven to be useful for Lotka-Voltera predator-preys systems [32], and it appears that they can be useful for more complex compartmental epidemic models as well [33] [34].

The main aim of the present paper is to show that our model has a unique endemic equilibrium which is globally asymptotically stable.

This SVEIR model could be used to assess the potential impact of an extended vaccination program (such as for the monovalent serogroup A conjugate MenVacAfric, an anti-meningococcal vaccine introduced in 2011 in Sub-saharan Africa), in order to compare with the impact of a pulse vaccination program.

In the next section, we present our SVEIR epidemic model. Section 3 presents some basic properties like the computation of the basic reproduction ration, $\mathcal{R}_{0}$, and such as the existence of the equilibrium points. In Section 4, we study the stability properties of the model and in Section 7, numerical simulations will be done with Cameroon data which deal with the vaccination campaign against polio. An conclusion round the paper.

\section{Model Description}

We divide the entire population into 5 sub-populations of epidemiological significance: susceptible, vaccinated, exposed, infective, and removed compartments with respective sizes $S, V, E, I$ and $R$. The latent compartment, $E$, takes into account the de- 
lay between the moment of the infection and the moment when an infected individual becomes infectious. The per capita death rates for susceptible, vaccinated, exposed, infective and recovered individuals are $d_{S}, d_{V}, d_{E}, d_{I}$ and $d_{R}$, respectively. The recruitment rate into the susceptible class is assumed to be constant and denoted by $\Lambda$. The per capita vaccination rate is $p$.

We assume mass action incidence $\beta S I$ for susceptible. Vaccination reduces the risk of infection by a factor $\theta \in(0,1)$. Thus, we have mass action incidence $\theta \beta V I$ for vaccinated individuals and the efficacy of the vaccine is $1-\theta$. The case $\theta=0$ corresponds to a perfect vaccine and the case $\theta=1$ corresponds to a vaccine with no effect. Each of these cases can be dealt with more simply and directly by studying the basic SEIR model.

The average duration of latency in class $E$ before progressing to class $I$ is $\frac{1}{\varepsilon}$, and the average time spent in class $I$ before recovery is $\frac{1}{\gamma}$. All parameters of the system are assumed to be positive.

Our model consists of the following system of ordinary differential equations:

$$
\left\{\begin{array}{l}
\dot{S}=\Lambda-\left(d_{S}+p\right) S-\beta S I \\
\dot{V}=p S-d_{V} V-\theta \beta V I \\
\dot{E}=\beta I(S+\theta V)-\left(d_{E}+\varepsilon\right) E \\
\dot{I}=\varepsilon E-\left(d_{I}+\gamma\right) I \\
\dot{R}=\gamma I-d_{R} R,
\end{array}\right.
$$

with initial conditions which satisfy $S(0), V(0), E(0), I(0), R(0) \geq 0$. A schematic of the model is shown in Figure 1.

Since $R$ does not appear in the equations for the other variables, we will consider the following system (model system (3) without the $R$ compartment):

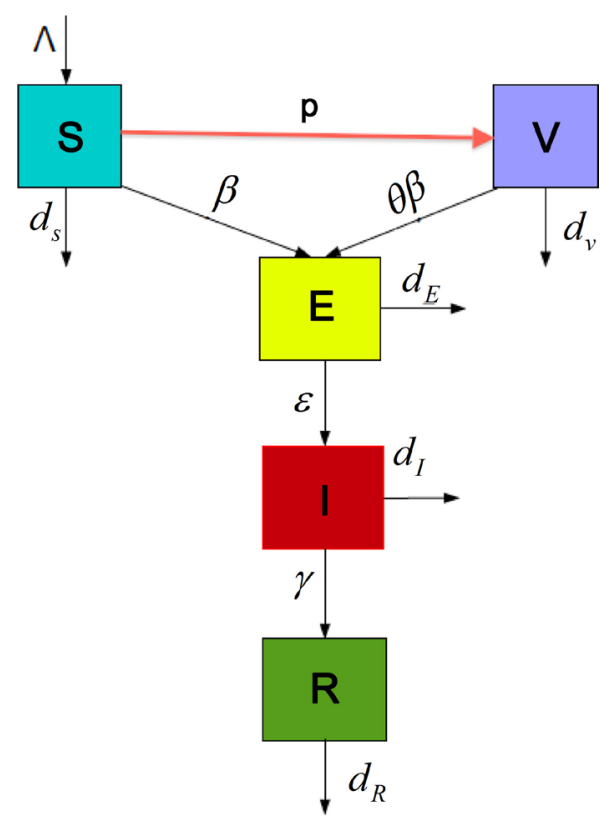

Figure 1. Schematic of the compartmental model. 


$$
\left\{\begin{array}{l}
\dot{S}=\Lambda-\left(d_{S}+p\right) S-\beta S I \\
\dot{V}=p S-d_{V} V-\theta \beta V I \\
\dot{E}=\beta I(S+\theta V)-\left(d_{E}+\varepsilon\right) E \\
\dot{I}=\varepsilon E-\left(d_{I}+\gamma\right) I
\end{array}\right.
$$

with initial conditions which satisfy $S(0), V(0), E(0), I(0) \geq 0$.

\section{Basic Properties and Equilibriums}

\subsection{A Compact Positively Invariant Absorbing Set}

In order that the model be well-posed, it is necessary that the state variables $S(t)$, $V(t), E(t)$ and $I(t)$ remain nonnegative for all $t \geq 0$. That is, the nonnegative orthant $\mathbb{R}_{+}^{4}$ must be positively invariant. Let

$$
\mathcal{K}=\left\{(S, V, E, I) \in \mathbb{R}_{+}^{4}: S+V+E+I \leq \frac{\Lambda}{d}, S \leq S^{*}, V \leq V^{*}\right\},
$$

where $d=\min \left\{d_{S}, d_{V}, d_{E}, d_{I}\right\}, S^{*}=\frac{\Lambda}{\left(d_{S}+p\right)}$ and $V^{*}=\frac{p S^{*}}{d_{V}}$.

Lemma 1. The compact set $\mathcal{K}$ is a positively invariant and attracting.

Proof. For each of the variables $S, V, E$ and $I$, when the variable is equal to zero, the derivative of that variable is non-negative in $\mathbb{R}_{+}^{4}$. It then follows from ([35], Proposition 2.1) that $\mathbb{R}_{+}^{4}$ is positively invariant.

Let $N=S+V+E+I$. Then

$$
\dot{N}=\Lambda-d_{S} S-d_{V} V-d_{E} E-d_{I} I \leq \Lambda-d N .
$$

Consequently,

$$
\liminf N(t) \leq \frac{\Lambda}{d}
$$

Similarly, $\dot{S}(t)<0$ when $S(t)>S^{*}$ and so

$$
\liminf S(t) \leq S^{*}
$$

Let $\epsilon>0$. Then for a given initial condition, there exists $T \geq 0$ such that $S(t) \leq S^{*}+\epsilon$ for all $t \geq T$. Then,

$$
\dot{V} \leq p\left(S^{*}+\epsilon\right)-d_{V} V
$$

for $t \geq T$. Thus,

$$
\liminf V \leq \frac{p S^{*}+\epsilon}{d_{V}} .
$$

This holds for all $\epsilon>0$ and so

$$
\liminf V \leq \frac{p S^{*}}{d_{V}}=V^{*} .
$$

Since $\mathcal{K}$ is a positively invariant absorbing set is sufficient to consider the dynamics of the flow generated by system (3) in $\mathcal{K}$.

It is easy to see that the model system (3) has a disease-free equilibrium 
$P^{*}=\left(S^{*}, V^{*}, E^{*}, I^{*}\right)$ given by

$$
S^{*}=\frac{\Lambda}{\left(d_{S}+p\right)}, V^{*}=\frac{p \Lambda}{d_{V}\left(d_{S}+p\right)}, E^{*}=0, I^{*}=0 .
$$

Additionally, an endemic equilibrium $\bar{P}$ may also exist.

\subsection{Basic Reproduction Ratio and Equilibrium}

Using the method of the references [36] [37], the basic reproduction number $\mathcal{R}_{\text {vac }}$ is

$$
\mathcal{R}_{\mathrm{vac}}=\frac{\varepsilon \beta\left(S^{*}+\theta \mathrm{V}^{*}\right)}{\left(d_{E}+\varepsilon\right)\left(d_{I}+\gamma\right)}
$$

Replacing $S^{*}$ and $V^{*}$ by their values in (9), we obtain:

$$
\mathcal{R}_{\mathrm{vac}}=\frac{\varepsilon \beta \Lambda}{\left(d_{S}+p\right)\left(d_{E}+\varepsilon\right)\left(d_{I}+\gamma\right)}\left(1+\frac{\theta p}{d_{V}}\right) .
$$

When there is no vaccination ( $p=0)$, system (3) is the standard SEIR model with

$$
\mathcal{R}_{0}=\left.\mathcal{R}_{\mathrm{vac}}\right|_{p=0}=\frac{\varepsilon \beta \Lambda}{d_{S}\left(d_{E}+\varepsilon\right)\left(d_{I}+\gamma\right)} .
$$

From Equation (10), we claim the following result.

Proposition 1. $\mathcal{R}_{\text {vac }} \leq \mathcal{R}_{0}$ if and only if $\theta d_{S} \leq d_{V}$.

Proof. It follows from (11) that

$$
\mathcal{R}_{\mathrm{vac}}=\frac{d_{V}+\theta p}{d_{V}} \frac{d_{S}}{d_{S}+p} \mathcal{R}_{0} .
$$

Thus, $\mathcal{R}_{\text {vac }} \leq \mathcal{R}_{0}$ is equivalent to

$$
\left(d_{V}+\theta p\right) d_{S} \leq d_{V}\left(d_{S}+p\right),
$$

from which the result follows.

The value of $\mathcal{R}_{\text {vac }}$ determines whether or not there exists an endemic equilibrium ([38], Theorem 2.3).

Theorem 1. If $\mathcal{R}_{\mathrm{vac}} \leq 1$, then there are no endemic equilibria. If $\mathcal{R}_{\mathrm{vac}}>1$, then there exists a unique endemic equilibrium $\bar{P}=(\bar{S}, \bar{V}, \bar{E}, \bar{I}))$.

(See Appendix for proof).

\section{Stability Analysis of Equilibriums}

\subsection{Stability Analysis of the DFE}

For local stability of the disease-free equilibrium, we claim the following:

Theorem 2. If $\mathcal{R}_{\mathrm{vac}}<1$, then the disease-free equilibrium is locally asymptotically stable and unstable if $\mathcal{R}_{\mathrm{vac}}>1$.

Proof. The Jacobian matrix of model (3) evaluate at the disease-free equilibrium is given by

$$
J\left(P^{*}\right)=\left(\begin{array}{cccc}
-\left(d_{S}+p\right) & 0 & 0 & -\beta S^{*} \\
p & -d_{V} & 0 & -\beta V^{*} \\
0 & 0 & -\left(d_{E}+\epsilon\right) & \beta\left(S^{*}+\theta V^{*}\right) \\
0 & 0 & \epsilon & -\left(d_{I}+\gamma\right)
\end{array}\right)
$$


The eigenvalues of $\mathcal{J}\left(P^{*}\right)$ are $\lambda_{1}=-\left(d_{S}+p\right), \lambda_{2}=-d_{V}$, and those of the following sub-matrices

$$
J_{1}=\left(\begin{array}{cc}
-\left(d_{E}+\epsilon\right) & \beta\left(S^{*}+\theta V^{*}\right) \\
\epsilon & -\left(d_{I}+\gamma\right)
\end{array}\right) .
$$

The characteristic polynomial of $J_{1}$ is given by

$$
\mathcal{P}_{1}(\lambda)=\lambda^{2}+\left(\gamma+\epsilon+d_{E}+d_{I}\right) \lambda+\left(1-R_{\mathrm{vac}}\right)\left(d_{E}+\epsilon\right)\left(\gamma+d_{I}\right)
$$

It clear that the roots of $\mathcal{P}_{1}$ have negative real parts if and only if $\mathcal{R}_{\text {vac }}<1$. It follows that the disease-free equilibrium $P^{*}$ is locally asymptotically stable whenever $\mathcal{R}_{\text {vac }}<1$ and unstable when $\mathcal{R}_{\mathrm{vac}}>1$. This end the proof.

The following result is proven in ([20], Theorem 4.1).

Theorem 3. If $\mathcal{R}_{\mathrm{vac}} \leq 1$, then the disease-free equilibrium is globally asymptotically stable.

If $\mathcal{R}_{\mathrm{vac}}>1$, then the disease-free equilibrium is unstable.

\subsection{Stability Analysis of the Endemic Equilibrium}

Our main result is the following theorem.

Theorem 4. If $\mathcal{R}_{\mathrm{vac}}>1$, then the endemic equilibrium point $(\bar{S} \bar{V}, \bar{E}, \bar{I})$ is globally asymptotically stable in $\mathbb{R}_{+}^{4}$.

Proof. Consider the following candidate Lyapunov function

$$
\mathcal{V}(S, V, E, I)=(S-\bar{S} \ln S)+(V-\bar{V} \ln V)+(E-\bar{E} \ln E)+\frac{d_{E}+\varepsilon}{\varepsilon}(I-\bar{I} \ln I) .
$$

Differentiating $\mathcal{V}(S, V, E, I)$ along solutions to (3) gives:

$$
\begin{aligned}
\dot{\mathcal{V}}= & d_{S} \bar{S}\left(2-\frac{S}{\bar{S}}-\frac{\bar{S}}{S}\right)+d_{V} \bar{V}\left(3-\frac{\bar{S}}{S}-\frac{V}{\bar{V}}-\frac{\bar{V}}{V} \frac{S}{\bar{S}}\right) \\
& +\beta \bar{I} \bar{S}\left(3-\frac{\bar{S}}{S}-\frac{I}{\bar{I}} \frac{S}{\bar{S}} \frac{\bar{E}}{E}-\frac{E}{\bar{E}} \frac{\bar{I}}{I}\right)+\theta \beta \overline{I V}\left(4-\frac{\bar{V}}{V} \frac{S}{\bar{S}}-\frac{I}{\bar{I}} \frac{V}{\bar{V}} \frac{\bar{E}}{E}-\frac{E}{\bar{E}} \frac{\bar{I}}{I}-\frac{\bar{S}}{S}\right) .
\end{aligned}
$$

Since

$$
\frac{S}{\bar{S}} \frac{\bar{S}}{S}=1 ; \frac{\bar{S}}{S} \frac{V}{\bar{V}} \frac{\bar{V}}{V} \frac{S}{\bar{S}}=1 ; \frac{\bar{S}}{S} \frac{I}{\bar{I}} \frac{S}{\bar{S}} \frac{\bar{E}}{E} \frac{E}{\bar{E}} \frac{\bar{I}}{I}=1 ; \frac{\bar{V}}{V} \frac{S}{\bar{S}} \bar{I} \frac{V}{\bar{I}} \frac{V}{\bar{V}} \frac{\bar{E}}{E} \frac{E}{\bar{E}} \frac{\bar{I}}{I} \frac{\bar{S}}{S}=1
$$

and,

$$
a_{1}+a_{2}+a_{3}+\cdots+a_{n} \geq n \sqrt[n]{a_{1} \cdot a_{2} \cdot a_{3} \cdots a_{n}}, \quad a_{1}, a_{2}, a_{3}, \cdots, a_{n} \geq 0
$$

Since arithmetical mean is greater than geometrical mean, we have the following inequalities

$$
\begin{aligned}
& 2-\frac{S}{\bar{S}}-\frac{\bar{S}}{S} \leq 0 \\
& 3-\frac{\bar{S}}{S}-\frac{V}{\bar{V}}-\frac{\bar{V}}{V} \frac{S}{\bar{S}} \leq 0 \\
& 3-\frac{\bar{S}}{S}-\frac{I}{\bar{I}} \frac{S}{\bar{S}} \frac{\bar{E}}{E}-\frac{E}{\bar{E}} \frac{\bar{I}}{I} \leq 0 \\
& 4-\frac{\bar{V}}{V} \frac{S}{\bar{S}}-\frac{I}{\bar{I}} \frac{V}{\bar{V}} \frac{\bar{E}}{E}-\frac{E}{\bar{E}} \frac{\bar{I}}{I}-\frac{\bar{S}}{S} \leq 0 .
\end{aligned}
$$


Therefore $\dot{\mathcal{V}} \leq 0$. Thank's to the direct Lyapunov theorem of stability, we conclude that $\bar{P}=(\bar{S} \bar{V}, \bar{E}, \bar{I})$ is stable.

It remain to prove that $\bar{P}=(\bar{S}, \bar{V}, \bar{E}, I)$ is asymptotically stable using the Lasalle invariance principle.

set

$$
\begin{aligned}
& A=2-\frac{S}{\bar{S}}-\frac{\bar{S}}{S} \\
& B=3-\frac{\bar{S}}{S}-\frac{V}{\bar{V}}-\frac{\bar{V}}{V} \frac{S}{\bar{S}} \\
& C=3-\frac{\bar{S}}{S}-\frac{I}{\bar{I}} \frac{S}{\bar{S}} \frac{\bar{E}}{E}-\frac{E \bar{I}}{\bar{E}} \frac{\bar{I}}{\bar{V}} \\
& D=4-\frac{S}{V} \frac{I}{\bar{S}}-\frac{V}{\bar{I}} \frac{V}{\bar{V}} \frac{\bar{E}}{E}-\frac{E}{\bar{E}} \frac{\bar{I}}{I}-\frac{\bar{S}}{S} .
\end{aligned}
$$

it's clear that;

$$
(\dot{\mathcal{V}}=0) \Leftrightarrow(A=B=C=D=0)
$$

Backing to the above relations, we have the following implications.

$$
\begin{gathered}
A=0 \Rightarrow \bar{S}=S \\
(S=\bar{S}, \quad B=0) \Rightarrow(V=\bar{V}) \\
(S=\bar{S}, C=0) \Rightarrow\left(\frac{I}{\bar{I}} \frac{\bar{E}}{E}=\frac{E}{\bar{E}} \frac{\bar{I}}{I}=1\right) \Rightarrow\left(\frac{E}{\bar{E}}=\frac{I}{\bar{I}}\right)
\end{gathered}
$$

If we set

$$
\frac{E}{\bar{E}}=\frac{I}{\bar{I}}=a \text {, then } E=a \bar{E} \text { and } I=a \bar{I}
$$

Finally we have,

$$
\dot{\mathcal{V}}(S, V, E, I)=0 \Leftrightarrow S=\bar{S}, \quad V=\bar{V}, \quad E=a \bar{E}, \quad I=a \bar{I}
$$

At the endemic equilibrium, we have

$$
\left\{\begin{array}{l}
\Lambda=\left(d_{S}+p\right) \bar{S}+\beta \overline{S I} \\
p \bar{S}=d_{V} \bar{V}+\theta \beta \overline{V I} \\
\beta \bar{I}(\bar{S}+\theta \bar{V})=\left(d_{E}+\varepsilon\right) \bar{E} \\
\varepsilon \bar{E}=\left(d_{I}+\gamma\right) \bar{I} .
\end{array}\right.
$$

Replacing $S, V, E, I$ by their values given by (24) in the second equation of system (25) yields

$$
0=p \bar{S}-d_{V} \bar{V}-\theta a \beta \bar{V} \bar{I} \Rightarrow p \bar{S}=d_{V} \bar{V}+\theta a \beta \overline{V I} .
$$

If we compare relation (26) with the last equation of (25), then we have:

$$
p \bar{S}=d_{V} \bar{V}+\theta \beta \overline{V I}, \quad \text { and } \quad a=1 .
$$

Consequently: $I=\bar{I}, E=\bar{E}$

Finally

$$
\dot{\mathcal{V}}(S, V, E, I)=0 \Leftrightarrow(S=\bar{S}, \quad V=\bar{V}, \quad E=\bar{E}, \quad I=\bar{I})
$$


Thus, the largest invariant set contained in $\{(S, V, E, I) \mid \dot{\mathcal{V}}=0\}$ is $\{\bar{P}=(\bar{S}, \bar{V}, \bar{E}, \bar{I})\}$.

Then the global stability of $\bar{P}=(\bar{S}, \bar{V}, \bar{E}, \bar{I})$ follows according to the Lasalle invariance principle [28].

\section{Numerical Simulations}

In this section we show via numerical simulations that when $\mathcal{R}_{\text {vac }}$ is lower than one (minor illness $\mathcal{R}_{\text {vac }}=0.70$ ), disease will be eliminated from the community, and when $\mathcal{R}_{\text {vac }}$ is greater than one (meningitis and paralytic form of polio), and epidemics will occurs or the disease will persists in the community. We explore also the impact of vaccination coverage in the spread of poliomyelitis.

\section{Parameters Description and Values}

Most of parameters values are from Cameroon, like natural rate of mortality. We assume that the natural rates of mortality of susceptible, recovered, exposed are the same. Value of vaccine efficacy, recovery rate and rate of apparition of clinical symptoms are coming from WHO. For vaccination coverage, we take different values in order to explore different situations. The recruitment rate of susceptible humans, $\Lambda$, likely is actually the birth rate, and are taken in [39] [40]. See Table 1 for the description of parameters and their based line or range value.

\section{Numerical Results and Interpretations}

Figure 2 illustrate the minor illness form of polio. We assume that $\gamma=0.5$, so $\mathcal{R}_{\text {vac }}=0.70$, and we have showed analytically that If $\mathcal{R}_{\text {vac }} \leq 1$, then the disease-free equilibrium is globally asymptotically stable. We see that in this case, healthy carriers and infectious tend toward horizontal axis, and the infection is eradicated after around 6 months.

In Figure 3, we are in the presence of the meningitis form of polio. Assuming that

Table 1. Description and values of parameters of model (3).

\begin{tabular}{ccc}
\hline Parameter & Description & Based line value or range \\
\hline$\Lambda$ & Recruitment rate of susceptible & 2.5 \\
$\beta$ & Effective contact rate & 0.1 \\
$1-\theta$ & Vaccine efficacy & 0.8 \\
$\varepsilon$ & Rate of development of clinical symptoms & $0.05-0.5$ \\
$\gamma$ & Recovery rate & 0.05 \\
$p$ & Vaccination coverage rate & {$[0,1]$} \\
$d_{S}$ & Natural mortality rate of susceptible & 0.0551 \\
$d_{V}$ & Natural mortality rate of vaccinated & 0.0551 \\
$d_{E}$ & Natural mortality rate of exposed & 0.0551 \\
$d_{I}$ & Mortality rate of infectious & 0.08 \\
$d_{R}$ & Natural mortality rate of recovered & 0.0551 \\
\hline
\end{tabular}


(a)

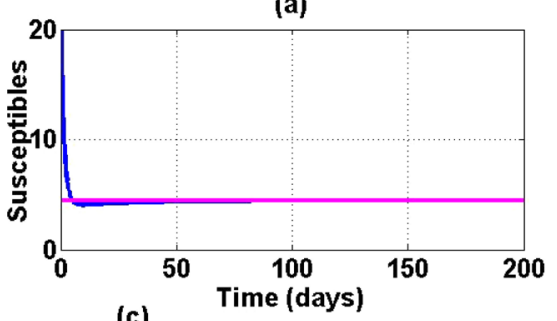

(c)

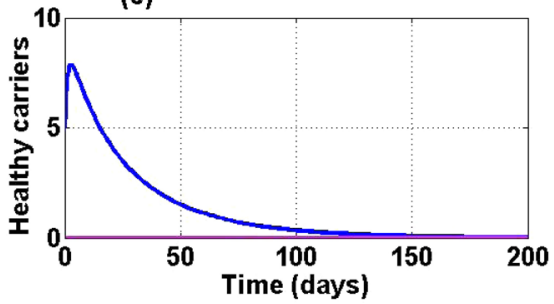

Figure 2. Minor illness $\mathcal{R}_{\text {vac }}=0.70$.

(a)
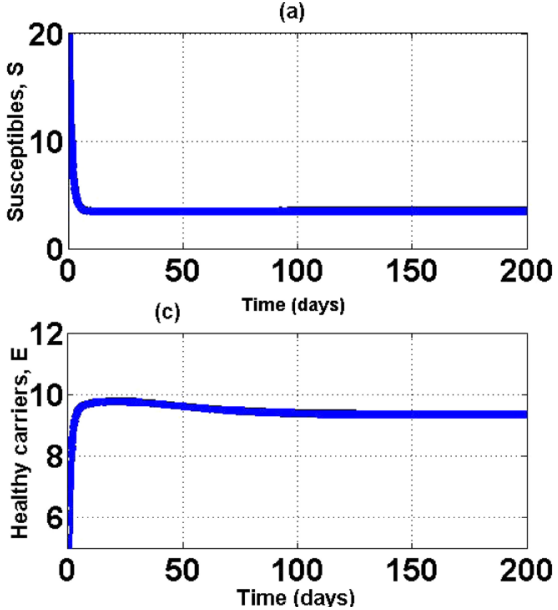

(b)

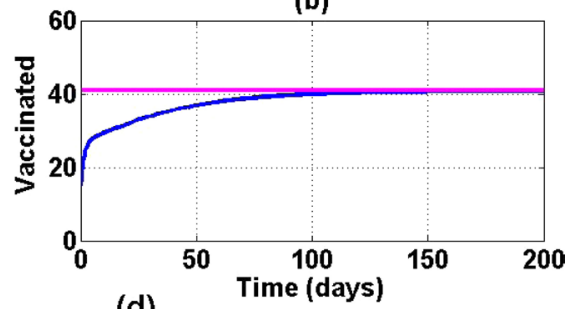

(d)

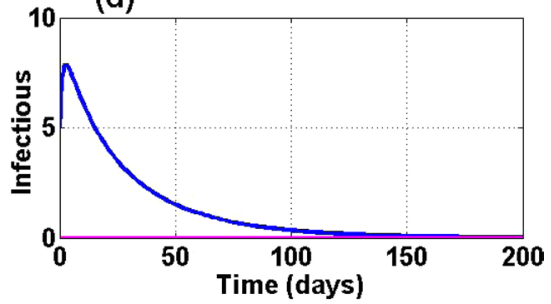

Figure 3. Meningitis form of polio $\mathcal{R}_{\mathrm{vac}}=1.46$.

$\gamma=0.2$ and vaccine coverage $p=0.8$, to have $\mathcal{R}_{\mathrm{vac}}=1.46$. It is clear that infection is a little more severe and the disease reaches at endemic equilibrium point and does not disappear.

In Figure 4, we are in the presence of the most severe form of polio: the paralytic form with $\gamma=0.005$, so $\mathcal{R}_{\mathrm{vac}}=3.15$. As in the case of meningitis form, the patient takes long time to heal and thus continue to transmit the infection during that time. It is important to note that remark is that the infection takes longer to reach the endemic equilibrium point and remains in the population despite vaccination.

We are in front of paralytic polio. We assume $\mathcal{R}_{\mathrm{vac}}=3.15$, and explore the effect of immunization on the dynamic of the disease. Figure 5 show that more vaccine coverage is high, the number of healthy carriers and infectious is low at equilibrium point. But it is noted that the infection remains in the population.

Figure 6, we explored three cases:

1) even if the vaccine is perfect and nobody is vaccinated; the infection is and remains high in the population $\theta=0$ and $p=0$;

2) The vaccination is made; even if the coverage is low infection decreases and reaches 
a an equilibrium point $\theta=0$ and $p=0.5$;

3) The last and not realistic situation is that infection is eradicated after one year, and when we have perfect vaccine and maximal vaccination coverage $\theta=0$ and $p=1$.

(a)

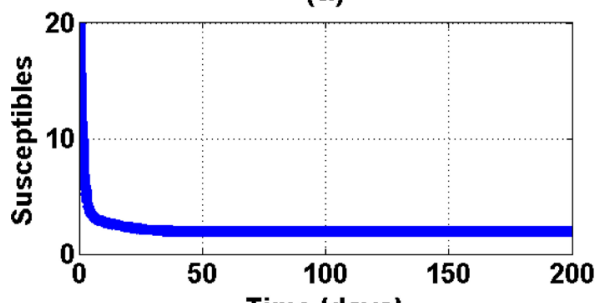

(c)

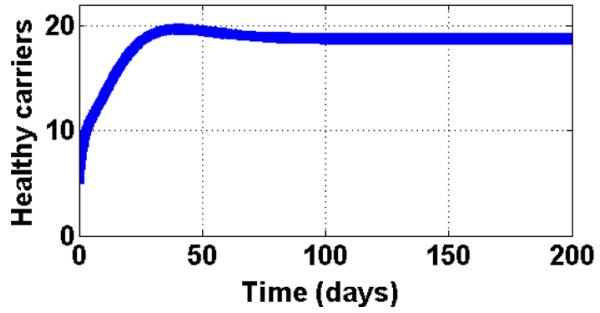

Figure 4. Paralytic form of polio $\mathcal{R}_{\mathrm{vac}}=3.15$.

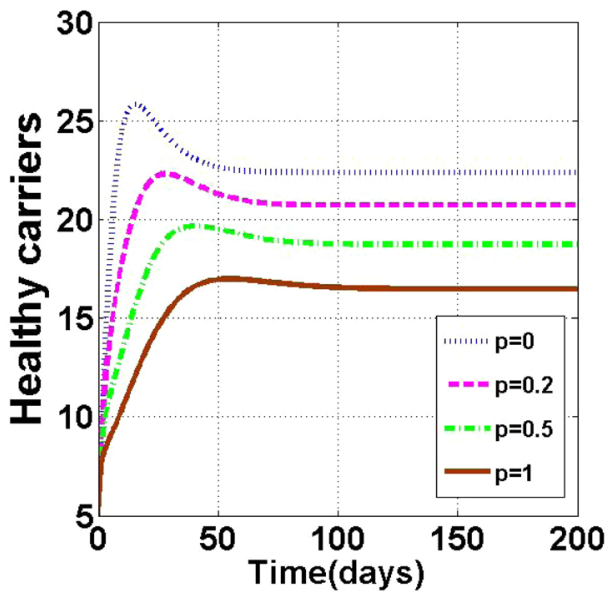

Figure 5. Impact of vaccine coverage.

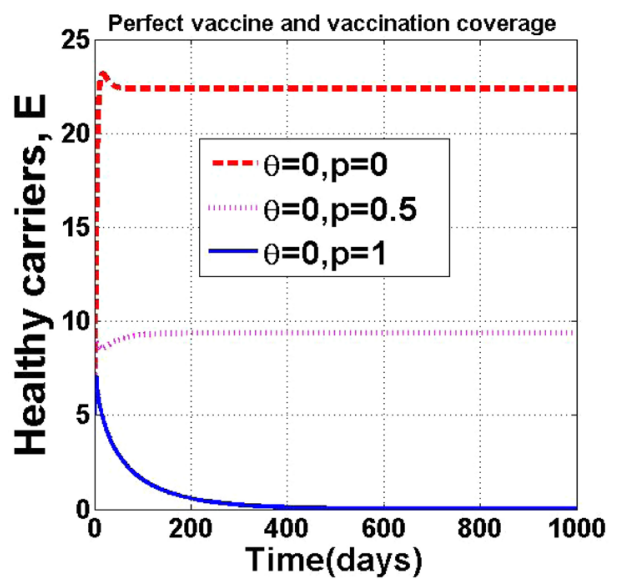

(b)

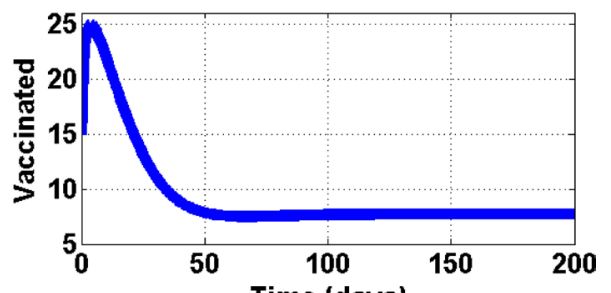

(d)
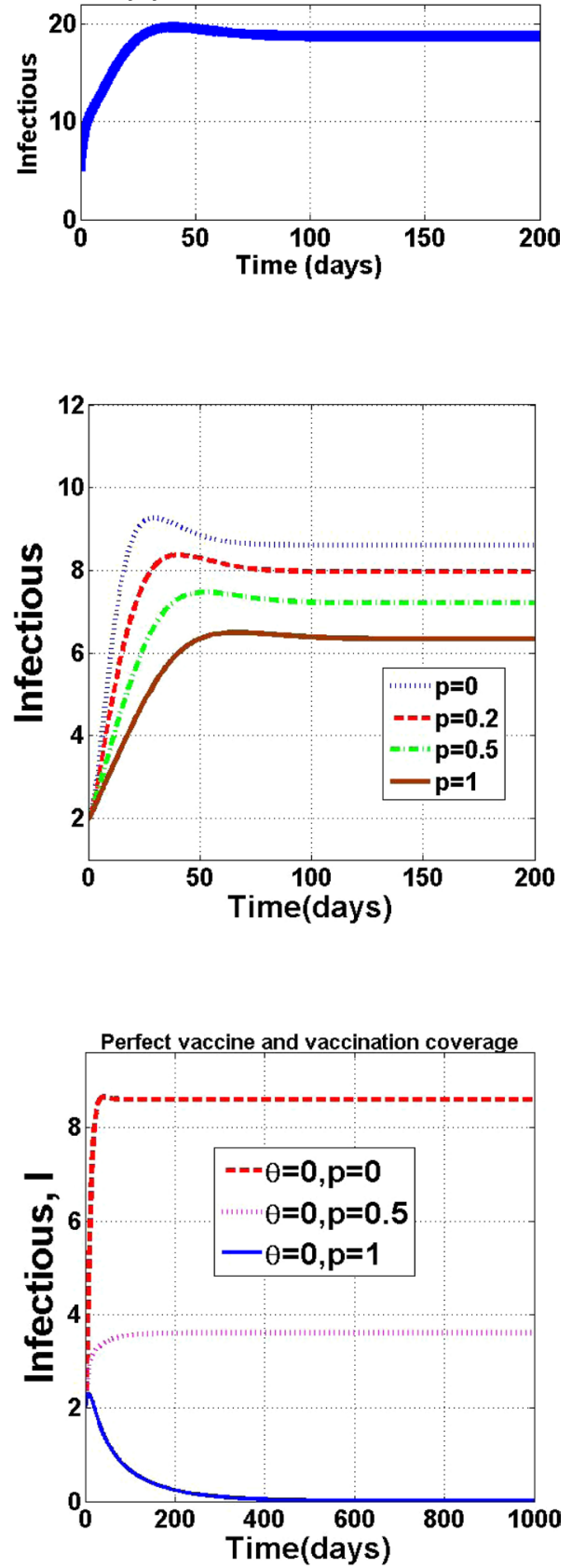

Figure 6. Impact of vaccine efficacy. 


\section{Conclusions}

We highlighted in this article the importance of vaccination in the control of the propagation of the poliomyelitis. We relied on the compartmentalized SVEIR model that characterizes the infectious diseases. We computed $\mathcal{R}_{\mathrm{vac}}$, key parameter related to the Reproduction, which governs the asymptotic behavior of the model. We then constructed a Lyapunov function to prove the global asymptotic stability of the endemic equilibrium whenever $\mathcal{R}_{\text {vac }}>1$.

Using data from AHALA (district of Yaound in Cameroon), we simulated the three different forms of polio namely the minor illness, the meningitis form and the paralytic form. In the case of minor illness of polio, we assumed that $\mathcal{R}_{\text {vac }}=0.70$. The model also allowed an endemic equilibrium point when $\mathcal{R}_{\text {vac }}$ is greater than 1 . In that case, we simulated both meningitis and paralytic form of polio, respectively with $\mathcal{R}_{\text {vac }}=1.46$ and $\mathcal{R}_{\text {vac }}=3.1$. We found that, the more the vaccine coverage is high, the more the healthy Carriers and Infectious are low. The simulations show that, to eradicate polio in the population means to have simultaneously a perfect vaccine and maximal vaccine coverage. Therefore, other control strategies are to be issued to finally reach that goal.

\section{Acknowledgements}

The first author acknowledges with thanks the High teacher Training College of Yaounde. H. A. would like to thank the Direction of the University Institute of Technology of Ngaoundere for their financial assistance in the context of research missions of September 13, 2016.

\section{References}

[1] World Health Organiation, Media Centre: Poliomyelitis, Fact Sheet. http://www.who.int/mediacentre/factsheets/fs114/en/\#

[2] Donaldson, L., El Sayed, N., Koplan, J., Nduati, R., Toole, M., Chowdhury, M., et al. (2012) Comité de suivi indépendant de l'initiative mondiale pour léradication de la poliomyélite.

[3] Centers for Disease Control and Prevention (CDC and Others), Follow-Up on Poliomyelitisunited States, Canada, Netherlands. 1979. MMWR. Morbidity and Mortality Weekly Report, 46, 1195.

[4] Miller, N.Z. (2004) The Polio Vaccine: A Critical Assessment of Its Arcane History, Efficacy, and Long-Term Health-Related Consequences. Medical Veritas, 1, 239-251.

[5] Sabin, A.B. (1984) Strategies for Elimination of Poliomyelitis in Different Parts of the World with Use of Oral Poliovirus Vaccine. Review of Infectious Diseases, 6, S391-S396.

[6] Miller, N.Z. (2004) The Polio Vaccine: A Critical Assessment of Its Arcane History, Efficacy, and Long-Term Health-Related Consequences. Medical Veritas, 1, 239-251.

[7] Centers for Disease Control and Prevention (CDC) and Others, The Pink book: Epidemiology and Prevention of Vaccine Preventable Diseases.

[8] A. A. of Pediatrics, et al. (1998) American Academy of Pediatrics. Committee on Infectious Diseases. Severe Invasive Group a Streptococcal Infections: A Subject Review. Pediatrics, $101,136$.

[9] Browne, C.J., Smith, R.J. and Bourouiba, L. (2015) From Regional Pulse Vaccination to Global Disease Eradication: Insights from a Mathematical Model of Poliomyelitis. Journal of Mathematical Biology, 71, 215-253.

[10] Tebbens, R., Pallansch, M.A., Kew, O.M., Cáceres, V.M., Sutter, R.W. and Thompson, K.M. 
(2005) A Dynamic Model of Poliomyelitis Outbreaks: Learning from the Past to Help Inform the Future. American Journal of Epidemiology, 162, 358-372. https://doi.org/10.1093/aje/kwi206

[11] Kalkowska, D.A., Tebbens, R., Pallansch, M.A., Cochi, S.L., Wassilak, S.G. and Thompson, K.M. (2015) Modeling Undetected Live Poliovirus Circulation after Apparent Interruption of Transmission: Implications for Surveillance and Vaccination. BMC Infectious Diseases, 15, 66. https://doi.org/10.1186/s12879-015-0791-5

[12] Bunimovich-Mendrazitsky, S. and Stone, L. (2005) Modeling Polio as a Disease of Development. Journal of Theoretical Biology, 237, 302-315. https://doi.org/10.1016/j.jtbi.2005.04.017

[13] Rahmandad, H., Hu, K., Tebbens, R.D. and Thompson, K. (2011) Development of an Individual-Based Model for Polioviruses: Implications of the Selection of Network Type and Outcome Metrics. Epidemiology and Infection, 139, 836-848.

https://doi.org/10.1017/S0950268810001676

[14] Gandica, I.D., Ocampo, L.M., Marín, E. and González, M. (2013) Model for the Vaccination against the Poliomyelitis. Journal of Mathematics and System Science, 3, 62-67.

[15] Thompson, J.E. (2014) A Class Structured Mathematical Model for Polio Virus in Nigeria.

[16] O’Reilly, K.M., Chauvin, C., Aylward, R.B., Maher, C., Okiror, S., Wolff, C., Nshmirimana, D., Donnelly, C.A. and Grassly, N.C. (2011) A Statistical Model of the International Spread of Wild Poliovirus in Africa Used to Predict And Prevent Outbreaks. PLoS Medicine, 8, e1001109. https://doi.org/10.1371/journal.pmed.1001109

[17] Wagner, B.G. and Earn, D.J. (2008) Circulating Vaccine Derived Polio Viruses and Their Impact on Global Polio Eradication. Bulletin of Mathematical Biology, 70, 253-280. https://doi.org/10.1007/s11538-007-9255-4

[18] Thompson, K.M., Duintjer, R., Tebbens, J. and Pallansch, M.A. (2006) Evaluation of Response Scenarios to Potential Polio Outbreaks Using Mathematical Models. Risk Analysis, 26, 1541-1556. https://doi.org/10.1111/j.1539-6924.2006.00843.x

[19] Hethcote, H.W. (2000) The Mathematics of Infectious Diseases. SIAM Review, 42, 599-653. https://doi.org/10.1137/S0036144500371907

[20] Gumel, A., McCluskey, C. and Watmough, J. (2006) An Sveir Model for Assessing Potential Impact of an Imperfect Anti-Sars Vaccine. Mathematical Biosciences and Engineering, 3, 485-512. https://doi.org/10.3934/mbe.2006.3.485

[21] Zou, L., Ruan, S. and Zhang, W. (2010) An Age-Structured Model for the Transmission Dynamics of Hepatitis B. SIAM Journal on Applied Mathematics, 70, 3121-3139. https://doi.org/10.1137/090777645

[22] Castillo-Chavez, C. and Song, B. (2004) Dynamical Models of Tuberculosis and Their Applications. Mathematical Biosciences and Engineering, 1, 361-404. https://doi.org/10.3934/mbe.2004.1.361

[23] Gumel, A., McCluskey, C.C. and Van den Driessche, P. (2006) Mathematical Study of a Staged Progression HIV Model with Imperfect Vaccine. Bulletin of Mathematical Biology, 68, 2105-2128. https://doi.org/10.1007/s11538-006-9095-7

[24] Sharomi, O., Podder, C., Gumel, A., Elbasha, E. and Watmough, J. (2007) Role of Incidence Function in Vaccine-Induced Backward Bifurcation in Some HIV Models. Mathematical Biosciences, 210, 436-463. https://doi.org/10.1016/j.mbs.2007.05.012

[25] Li, M.Y. and Muldowney, J.S. (1995) Global Stability for the Seir Model in Epidemiology. Mathematical Biosciences, 125, 155-164. https://doi.org/10.1016/0025-5564(95)92756-5

[26] Muldowney, J.S. (1990) Compound Matrices and Ordinary Differential Equations. Rocky Mountain Journal of Mathematics, 20, 857-872. https://doi.org/10.1216/rmjm/1181073047

[27] Lakshmikantham, V. and Leela, S. (1969) Differential and Integral Inequalities. Academic 
Press, Cambridge.

[28] LaSalle, J.P. (1976) The Stability of Dynamical Systems. Vol. 25, SIAM. https://doi.org/10.1137/1.9781611970432

[29] Wei, H., Jiang, Y., Song, X., Su, G. and Qiu, S. (2009) Global Attractivity and Permanence of a SVEIR Epidemic Model with Pulse Vaccination and Time Delay. Journal of Computational and Applied Mathematics, 229, 302-312. https://doi.org/10.1016/j.cam.2008.10.046

[30] Jiang, Y., Wei, H., Song, X., Mei, L., Su, G. and Qiu, S. (2009) Global Attractivity and Permanence of a Delayed SVEIR Epidemic Model with Pulse Vaccination and Saturation Incidence. Applied Mathematics and Computation, 213, 312-321. https://doi.org/10.1016/j.amc.2009.03.024

[31] Hyman, J. and Li, J. (2005) Differential Susceptibility Epidemic Models. Journal of Mathematical Biology, 50, 626-644. https://doi.org/10.1007/s00285-004-0301-7

[32] Beretta, E. and Takeuchi, Y. (1988) Global Asymptotic Stability of Lotka-Volterra Diffusion Models with Continuous Time Delay. SIAM Journal on Applied Mathematics, 48, 627-651. https://doi.org/10.1137/0148035

[33] Fall, A., Iggidr, A., Sallet, G. and Tewa, J.J. (2007) Epidemiological Models and Lyapunov Functions. Mathematical Modelling of Natural Phenomena, 2, 55-73. https://doi.org/10.1051/mmnp:2008011

[34] Thieme, H. (2011) Global Stability of the Endemic Equilibrium in Infinite Dimension: Lyapunov Functions and Positive Operators. Journal of Differential Equations, 250, 37723801. https://doi.org/10.1016/j.jde.2011.01.007

[35] Haddad, W.M., Chellaboina, V. and Hui, Q. (2010) Nonnegative and Compartmental Dynamical Systems. Princeton University Press, Princeton.

[36] Diekmann, O. and Heesterbeek, J. (2000) Mathematical Epidemiology of Infectious Diseases: Model Building, Analysis, and Interpretation. Wiley, Hoboken.

[37] Van den Driessche, P. and Watmough, J. (2002) Reproduction Numbers and Sub-Threshold Endemic Equilibria for Compartmental Models of Disease Transmission. Mathematical Biosciences, 180, 29-48. https://doi.org/10.1016/S0025-5564(02)00108-6

[38] Bonzi, B., Fall, A., Iggidr, A. and Sallet, G. (2011) Stability of Differential Susceptibility and Infectivity Epidemic Models. Journal of Mathematical Biology, 62, 39-64. https://doi.org/10.1007/s00285-010-0327-y

[39] Abboubakar, H., Kamgang, J.C., Nkamba, L.N., Tieudjo, D. and Emini, L. (2015) Modeling the Dynamics of Arboviral Diseases with Vaccination Perspective. Biomath, 4, Article ID: 1507241. https://doi.org/10.11145/j.biomath.2015.07.241

[40] Abboubakar, H., Kamgang, J.C. and Tieudjo, D. (2016) Backward Bifurcation and Control in Transmission Dynamics of Arboviral Diseases. Mathematical Biosciences, 278, 100-129. http://www.sciencedirect.com/science/article/pii/S0025556416300633 https://doi.org/10.1016/j.mbs.2016.06.002 


\section{Appendix}

\section{Proof of Theorem 1}

Proof. In order to determine the existence of possible endemic equilibrium, that is, equilibrium with all positive components which we denote by

$$
P=(\bar{S}, \bar{V}, \bar{E}, \bar{I}),
$$

we have to look for the solution of the algebraic system of equations obtained by equating the right hand sides of system (3) to zero. In this way we obtain the implicit system of equations,

$$
\bar{S}=\frac{\Lambda}{d_{S}+p+\beta \bar{I}}, \quad \bar{V}=\frac{p \bar{S}}{d_{V}+\theta \beta \bar{I}}, \quad \bar{E}=\frac{d_{I}+\gamma}{\epsilon} \bar{I},
$$

where $\bar{I}$ is solution of the following equation

$$
A_{1} \bar{I}^{2}+A_{2} \bar{I}+A_{3}=0
$$

with $A_{1}=-\mathcal{R}_{\text {vac }}^{2} \theta d_{V}\left(\epsilon+d_{E}\right)^{3}\left(d_{S}+p\right)\left(\gamma+d_{I}\right)^{3}$,

$$
A_{2}=-\mathcal{R}_{\text {vac }} \epsilon \Lambda\left(\gamma+d_{I}\right)^{2}\left(\epsilon+d_{E}\right)^{2}\left[d_{V}^{2}+p \theta\left(d_{V}+d_{S} \theta+p \theta\right)+\theta d_{V}\left(d_{S}+p\right)\left(1-\mathcal{R}_{\mathrm{vac}}\right)\right],
$$

and $A_{3}=\epsilon^{2} \Lambda^{2}\left(\epsilon+d_{E}\right)\left(d_{V}+p \theta\right)^{2}\left(\gamma+d_{I}\right)\left(\mathcal{R}_{\mathrm{vac}}-1\right)$.

Note that coefficient $A_{1}$ is always negative and coefficient $A_{3}$ is positive (resp. negative) if and only if $\mathcal{R}_{\text {vac }}$ is greater (less) than unity. Thus, model system (3) admits only one endemic equilibrium whenever the basic reproduction number is greater than unity. When $\mathcal{R}_{\text {vac }} \leq 1$, we have $A_{2}$ negative. It follows that the model system (3) does not have any endemic equilibrium point whenever $\mathcal{R}_{\mathrm{vac}} \leq 1$.

Submit or recommend next manuscript to SCIRP and we will provide best service for you:

Accepting pre-submission inquiries through Email, Facebook, LinkedIn, Twitter, etc. A wide selection of journals (inclusive of 9 subjects, more than 200 journals)

Providing 24-hour high-quality service

User-friendly online submission system

Fair and swift peer-review system

Efficient typesetting and proofreading procedure

Display of the result of downloads and visits, as well as the number of cited articles

Maximum dissemination of your research work

Submit your manuscript at: http://papersubmission.scirp.org/

Or contact ojmsi@scirp.org 